\section{The effect of capsicum frutescens-I to transient receptor potential vinaloid-1, toll like receptors (tlr-4) and interleukin 1 beta (il-1 $\beta$ ) on periodontitis}

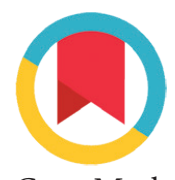

CrossMark

\author{
Jenny Sunariani, ${ }^{1 *}$ Latief Mooduto, ${ }^{2}$ Yuliati ${ }^{1}$
}

\title{
Abstract
}

Objective: Indonesia has many kinds of useful herbs, which are often used as species such as chili, pepper or cayenne pepper (capsicum frutescens L). Previous study showed topical capsaicin can be used as a therapy to cure pain due to inflammation. Small concentrations of capsaicin can attenuate cytokines in the inflammatory process. Capsaicin studies in animal model showed activation of the transient receptor potential vanilloid-1. Capsaicin can decrease various cytokines such as IL-6, IL-12, IL-1ß and increase IL-10. Capsaicin is a natural agonist for transient receptor potential vanilloid-1. The aim of this study is to prove the effect of capsaicin on transient receptor potential vanilloid- 1 expression and TNF- $a$ and TLR-4 toward pain.

Material and Methods: An experimental laboratory research used animal model Wistar male rats (rattus novegicus) induced with
Aggregatibacter actinomycetemcomitans serotype $b$ and green chili extract (capsicum frutescens L.) with a dose of $0.0912 \mathrm{mg} / \mathrm{kg} / \mathrm{day}$ was applied to surface of the gingiva on maxillary first molar for 7 days. An immunohistochemical examination was conducted to see the density of transient receptor potential vanilloid-1 and the expression of TLR-4 and IL-1 $\beta$ in the mucosal tissues of the oral cavity.

Results: There were significant differences in the applications of Capsicum frutescens $L$. with increasing of TRPV ligand-1 and IL-1 $1 \beta$ $(p<0.05)$, while the TLR-4 $(p>0.05)$ showed a significant difference to TRPV- 1 and IL-1 $\beta$. There is no significant difference to TLR-4.

Conclusion: Capsaicin can increase TRPV- 1 and decrease IL-1 $\beta$ but did not affect the TLR-4. Capsaicin can be used to decrease pain and accelerate healing process in periodontitis.
'Department of Oral Biology, Faculty of Dental Medicine, Universitas Airlangga, Surabaya, Indonesia

${ }^{2}$ Department of Conservative Dentistry, Faculty of Dental Medicine, Universitas Airlangga, Surabaya, Indonesia
*Correspondence to: Jenny Sunariani, Department of Oral Biology, Faculty of Dental Medicine, Universitas Airlangga, Surabaya, Indonesia jenny-s@fkg.unair.ac.id

Received: 10 January 2017 Revised: 25 May 2017 Accepted: 21 June 2017 Available Online: 01 August 2017

Keywords: Capsaicin, Inflammation, transient receptor potential vanilloid-1, IL-1ß, TLR-4

Cite this Article: Sunariani J, Mooduto L, Yuliati. 2017. The effect of capsicum frutescens-I to transient receptor potential vinaloid-1, toll like receptors (tlr-4) and interleukin 1 beta (il-1ß) on periodontitis. Journal of Dentomaxillofacial Science 2(2): 114-118. D0I: 10.15562/jdmfs.v2i2.527

\section{Introduction}

Herbal ingredients are widely used in Indonesia to cure diseases. Nowadays, people prefer natural than synthetic materials to use as therapeutic agents because of the adverse effects of synthetic materials. Indonesia has many kinds of useful herbs; one of them that is often used is from a species of chili pepper or cayenne pepper. $^{1}$ Cayenne pepper (capsicum frutescens L) is a herbaceous plant with a height of $50-100 \mathrm{~cm}$ and with a lot of branches on the trunk. People can use the fruit as a condiment, as a mix seasoning to add in food, and as traditional medicine. Fruit of capsicum frutescens L. has a powerful stimulant effect on the heart and peripheral blood flow, antirheumatic, anticoagulants and antithrombotic. Those effects are mostly caused by capsaicin, the active ingredient contained in the fruit stem of capsicum frutescens L. Previous research used capsaicin topically showed that it can be used as a therapy to cure pain due to inflammation. ${ }^{2}$ The capsaicin content of each varieties of chili in Bandung showed that green chili (capsicum frutescens L.) has the highest amount of capsaicin that is equal to $2.11 \%{ }^{3}$ The capsaicin has currently been used as a study material to determine the mechanism of pain and in pharmacotherapy to treat several pain disorders. Some other researches also show that capsaicin inhibits adhesion, growth, colonization and biofilm formation of gramnegative bacteria. ${ }^{4}$ The anti-inflammatory potential of capsaicin is supported by the results of research using small concentrations of capsaicin attenuating cytokines in the systemic inflammatory process. ${ }^{5,6}$ Animals were given $2 \mathrm{ml}$ of capsaicin with minimum inhibitory concentration (MIC) of $16 \mathrm{mg} / \mathrm{L}$ and the results showed via activation of TRPV-1, Tumor Necrosis Factor Alpha (TNFa), interleukin 6 (IL-6), interleukin 12 (IL-12). Interleukin 1 beta (IL-1 $\beta$ ) decreased after treatment with capsaicin, whereas Interleukin 10 (IL-10), which is an anti-inflammatory cytokines increased. ${ }^{5}$ The anti-inflammatory mechanism of capsaicin is characterized by the sensation of heat which will further stimulate cessation of inflammatory mediator release. The capsaicin is a natural agonist for transient receptor potential vanilloid-1 1 (TRPV-1) that will induce intracellular signaling in nerve cells through the vanilloid receptor. ${ }^{7}$ In the state of infection with fever symptoms, senses of 
taste buds will be affected. ${ }^{8}$ The primary cause of periodontitis is infections due to gram-negative A. actinomycetem-comitans in the oral cavity that will release the LPS binding to the TLR4 receptor. This inflammation will be processed in the cell and will release several proinflammatory cytokines including TNF $\alpha$, IL-1 $\beta$ and MMP8.

\section{Material and Methods}

This was an experimental laboratory research that used the gingival tissue of strain wistar rats. Inclusion criteria of the experimental animals were 21 strains Wistar male rats divided into two treatment groups and one control group. Animals had to be physically healthy, aged 8-12 weeks with a weight of 150-250 grams. Male rats were selected because they were not affected by hormonal and pregnancy, while the selected age were 8-12 weeks because the rats were still in young adulthood age and the immunological response would be quickly visible. The number of samples was determined using Lemeshow et al. ${ }^{9}$

Variables in this study included: the independent variable was the extract of cayenne pepper, the dependent variable was the number of inflammatory cells and control variables included age, gender. body weight of the wistar, A. actinomycetemcomitans serotype b concentrations, nutrition, maintenance and sanitation, cage and treatment time for the wistar.

The research was conducted in the Laboratory of Microbiology Faculty of Dentistry Airlangga University, Laboratory of Biochemistry Medical Faculty Airlangga University, Laboratory of Phytochemistry Pharmacy Airlangga University and Laboratory of Pathology Anatomy Medical Faculty Airlangga University and Laboratory of Physiology Medical Faculty Brawijaya University.

\section{Extraction of cayenne pepper}

Separation of the capsaicin compound from chili was done using soxhlet extraction method. ${ }^{5}$ Before extracted, samples were dried in an oven with a temperature of $60^{\circ} \mathrm{C}$. Then samples were crushed using a blender to obtain samples of fine powder. Extraction was done with ethanol for 8 hours to obtain a reddish brown extract. The extract was concentrated using a rotary evaporator in order to produce dark brown reddish concentrated extracts in gel form.

\section{Preparation of Bacteria}

Aggregatibacter actinomycetemcomitans serotype b bacteria were inoculated in AAGM media or LB media and then incubated for 24 hours at $37^{\circ} \mathrm{C}$.
After that, culture with bacterial density of 108 CFU was made. The samples groups were divided as follows; Negative control group: rats were induced using aqua Dest and given $0.9 \% \mathrm{NaCl}$ solvent for 7 days. Positive control group: rats were induced with A. Actinomycetemcomitans serotype b. bacteria once a day for 7 days.

In treatment 1 group: rats were induced with $\mathrm{A}$. Actinomycetemcomitans serotype b bacteria once a day for 7 days and were given extracts of green cayenne pepper with a dose of $0.0912 \mathrm{mg} / \mathrm{kg} /$ day which was applied on the surface of the maxillary first molar gingiva.

\section{Treatment of the samples}

Aggregatibacter actinomycetemcomitans serotype b were induced in the Wistar's cervical portion of the maxillary first molar with the density of $108 \mathrm{CFU}$, which was given at least once a day for 7 days to get symptoms of aggressive periodontitis. ${ }^{11,12}$ Extract of cayenne pepper was given after the induction of $\mathrm{A}$. actinomycetemcomitans serotype $\mathrm{b}$ bacteria once a day for 7 days. $^{2}$ The extract of cayenne pepper was applied topically in maxillary first molar gingiva of the Wistar rats. Immunohistochemistry (IHC) calculation method was done for estimating the density of TRPV 1 over the expression of TLR- 4 and IL- $1 \beta$ in the oral mucosal tissue of rats. There are 21 slides that consisted of 7 slide $x 3$ group examination. Each tissue sample was made into a slice with a thickness of $4 \mu \mathrm{m}$ and then detected for IHC examination to observe the distribution of immunocompetent cells. For the purposes of the calculation, slides were closed and given a new random number, so that the examiner did not divide the examined slides based on a particular sample group (blind). This examination were done by two examiners; each one was operator 1 and operator 2 (researchers). Inspection and sample calculations were performed separately by the two examiners. Each slide was observed with a magnification of $1000 \mathrm{x}$ and 5 visual field. ${ }^{13,14}$ The result of each calculation was written on worksheets and the average value per visual field was taken. The IHC was used for counting the numbers of immunocompetent cells based on structural model. Statistic analyses were done when all results have been returned to the actual code. In order to guarantee the representation and to reduce errors in the results, observation was done for 20 field of view with 1000x magnification that contained approximately 1500 s. 
Immunohistochemistry Result.

TRPV-1 Expression

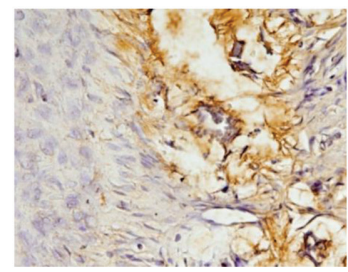

Negative Control

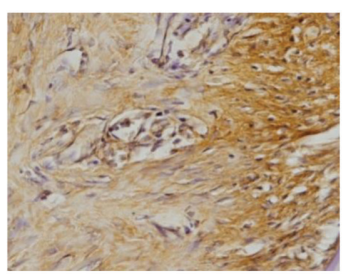

Positive Control

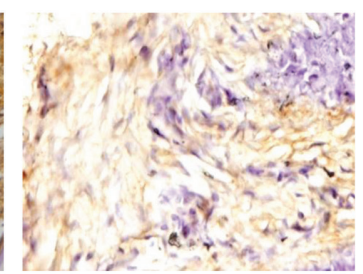

A. actinomycetemcomitans + C. frutescens L.

Figure 1 TRPV-1 expression using Immunohistochemistry staining on capsicum FL application day-7 (400x). Results of lymphocytes number expressing TRPV1 were obtained in average value: the treatment group was $15.87 \pm 1.96$ and the control group was $23.33 \pm 2.06$; On the 14th day of healing: the treatment group was $17.83 \pm 2.13$ and the control group was $31.00 \pm 0.89$. The t-test result with $\alpha=0.05$ indicates that there was a difference in the number of fibroblasts between control and treatment group

IL1- $\beta$ Expression

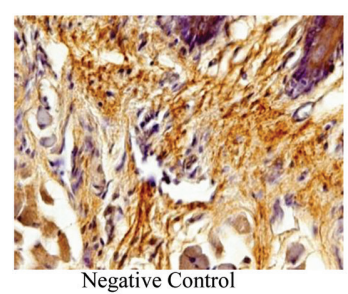

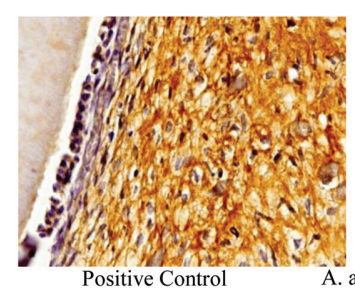

Positive Control

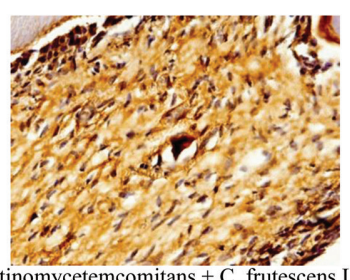

actinomycetemcomitans + C. frutescens $L$.

Figure 2 IL1- $\beta$ expression using immunohistochemistry staining on C. frutescens L application day-7 (400x)

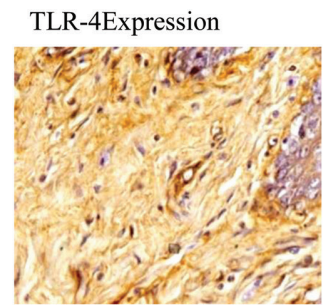

Negative Control

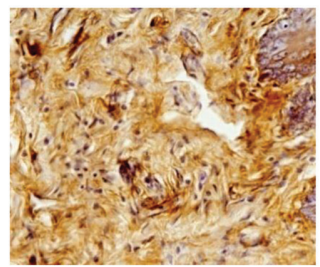

Positive Control

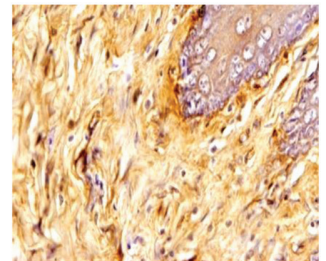

A. actinomycetemcomitans + C. frutescens L.

Figure 3 Expression of TLR-4 using immunohistochemistry staining on C. frutescens L application day-7 (400x)

were induced on the Wistar's cervical portion of the maxillary first molar for 7 days until periodontitis was existed. Treatment group was given cayenne pepper extract after the induction of A. Actinomycetemcomitans bacteria serotype b. Rats were anesthetized according to their body weight (30 $\mathrm{mg} / \mathrm{kg}$ thionembutal) and then being sacrificed for the gingival tissue sampling in the induction area to make preparations samples. Specimen contained tooth sockets, which were fixated in $10 \%$ formalin for 24 hours, decalcified with EDTA, then followed by a histologic procedure to make paraffin block, so that the gingiva could be cut longitudinally with a semi-serial thinness of $4 \mu \mathrm{m}$ to make microscopic

preparation. A microscopic preparation examination was done by counting the number of cells expressing TRPV-1, TLR- 4 and IL-1 $\beta$ by immunohistochemical staining then the data was analyzed.

Expression of TRPV1, TLR- 4 and IL- $1 \beta$ by Immunohistochemistry method using immunoRatio analysis is shown in figure 1,2 and 3.

\section{Discussion}

The capsicum frutescens is a vegetable used daily and the substance capsaicin (8-methyl-N-6-vanillyl nonenamide) is responsible for its hot and spicy nature of the vegetable, known as an additional flavor and sought after in gastronomy. Capsaicin and several related molecules are known by the collective name capsaicinoids and they are produced by all plants of the genus capsicum, with the exception of the bell pepper (capsicum annuum), which produces no capsaicin. Because capsaicin is not water soluble, alcohols and other organic solvents are used to solubilize capsaicin in topical preparations and sprays. This liposolubility is likely to explain why an oral excess of capsaicin in food is not alleviated by drinking water, whereas a yogurt-based drink, such as a lassi, is able to remove the vanilloid from the mouth. Capsaicin can produce a number of pain-related effects that depend on the dose and route of administration. The consequent effects may be sensitization, desensitization, withdrawal of afferent nerve terminals, or even avert death of afferents when given to neonatal animals. ${ }^{15}$ The stimulation from the outside stimuli either caused by germs, high intensity mechanical or chemical stimuli, can cause pain. Pain is a sign of damage in the body, both in the skin tissue and or visceral mucosa.

The TRPV1 receptor is a nonselective ligandgated cation channel. It is an integrator of many physical and chemical stimuli, including capsaicin and noxious heat $\left(>43^{\circ} \mathrm{C}\right)$, as well as being activated by protons $(\mathrm{pH}<5.2)$, endogenous lipids and certain inflammatory mediators. ${ }^{16}$ All compounds are lipophilic and therefore act at intracellular binding. ${ }^{17}$ Stimuli are detected and transduced through opening of the ion channel, which results in entry of cations such as $\mathrm{Na}^{+}$and $\mathrm{Ca}^{2+}$ in to the neuron; although the channel is nonselective, it has been shown to have a high preference for $\mathrm{Ca}^{2+} .{ }^{15}$ In this study, capsaicin was applied in the mucosal of Wistar rats with infections of A. actinomycetem- 
increase of capsaicin stimulation activate TRPV-1 ligand. This ligand receptor regulates pain and inflammation associated signals. It is expressed on immune cells and sensory neurons; there by the application of capsaicin will increase TRPV-1 receptor response.

TRPV-1 known as capsaicin receptor is a part of the ion channel transient release potential (TRP) that has an important role in helping the body sensing hot or warm..$^{18}$ Capsaicin activates TRPV-1 and enter the long refractory state that causes the excited nerves to become resistant to various stimuli, ranging from mechanical stress to endogenous or exogenous pain and proinflammatory substances. After the excitation, the TRPV-1 releases sensory neuropeptides which depends on the concentration of capsaicin used. ${ }^{19}$ Capsaicin results nociceptive fibers experience "defunctionalization" that brings out analgesic effect. Defunctionalization includes loss of membrane potential, reduces neuropeptides, transports inability of neurotrophic factors, is reversible retraction of epidermal and dermal nerve fibers terminal. ${ }^{7}$ Therefore, the effect of capsaicin will increase the TRPV-1 receptor response and cause higher analgesic effect.

Disruption of periodontal tissue homeostasis has the potential to cause the initiation and progression of periodontitis. ${ }^{20}$ One limitation of previous study is the lack of information on the possible involvement of TRPV1 in the pathogenesis of periodontal diseases. A recent study using immunohistochemistry showed that TRPV1 expression and distribution differed in subgingival specimens from patients with periodontitis and healthy individuals. ${ }^{21}$ However, further studies using both immunohistochemical and other biological methods are required. A more thorough understanding of the mechanism of action of capsaicin in GEC biology can potentially lead to the development of new therapeutic approaches for periodontal diseases.

\section{Capsaicin effect on TLR-4}

The study results showed no significant difference from the application of capsaicin to TLR-4, which means the application of capsaicin did not affect the TLR-4 receptor. This was because capsaicin was not the ligand of TLR-4 receptor so it had no effect and the TLR-4 expression remained high.

TLR4 functions as pattern recognition receptors for innate immune detection of tissue levels of bacterial-derived factors such as LPS. ${ }^{22}$ The finding show that capsaicin-sensitive nociceptors (i.e., TRPV1-positive) expressing TLR4 is consistent with the hypothesis that LPS can sensitize nociceptors via direct activation of this receptor. Therefore, previous study evaluated whether LPS derived from P. gingivalis directly sensitized these neurons. They found that LPS did not activate these neurons, but significantly sensitized them to an enhanced response to a chemical stimulus. Thus, LPS is capable of directly interacting with capsaicin-sensitive neurons, leading to exaggerated responses to noxious stimuli. ${ }^{23}$ The TLR is important in the mechanism of innate immunity and serves as a sensor of the pathogen germs existence. The TLR-4 is expressed on CD4+ $\mathrm{T}$ cells during its unclear function. Bacteria will release LPS which will be known by the receptor TLR- 4 and cause signaling in cells. ${ }^{24}$ Inflammation will be proceeded by the fibroblast cells, which then continuously releases several proinflammatory cytokines. Application of capsaicin does not affect the TLR-4 receptor so that the inflammatory process will continue to take place and the products of proinflammatory cytokines such as TNF- $\alpha$ and IL $1 \beta$ remains high.

\section{Capsaicin effect on IL-1 $\beta$ expression}

The results of this study showed that there was significant difference on the application of capsaicin to the expression of IL- $1 \beta$. In other words, capsaicin suppresses the expression of IL- $1 \beta$ due to its stimulation that will cause TRPV-1 induction, which is associated with inflammation besides the pain; hence, inflammatory response is activated in the form of decreased expression of proinflammatory cytokine IL- $1 \beta$.

The IL- $1 \beta$ is a chemical substance that plays an important role in the immune system and is a mediator of inflammatory response to infection. The IL- $1 \beta$ is synthesized as a form of protein after stimulation. The expression of IL- $1 \beta$ is induced by the transcription factor NF- $\mathrm{kB}$ due to an exposure by innate immune. The exposure of LPS in macrophages and dendritic cells binds to TLR- 4 and act as pathogen-associated molecular pattern. The synthesis of precursor IL- $1 \beta$ was also induced by the stimulation of innate immune system by Tolllike receptors (TLRs) or RIG-like receptors (RLRs). With the application of capsaicin, it induced TRPV-1 resulting in decreased expression of IL- $1 \beta$.

Periodontal diseases are inflammatory diseases in which microbial etiologic factors induce a series of host responses that mediate inflammatory events. In susceptible individuals, dysregulation of inflammatory and immune pathways leads to chronic inflammation, tissue destruction, and disease. ${ }^{23}$ Periodontitis is an inflammatory disease that causes an increase of proinflammatory cytokines that plays a role in inflammatory processes associated with destruction of the periodontium tissue. ${ }^{24,25}$ Some cytokines act as markers in the presence or absence of periodontal disease. Cytokines play an important 
role in the inflammatory process. ${ }^{26} \mathrm{IL}-1 \beta$ and IL-6 is produced locally in the damaged periodontium tissue and moves together with GCF (Gingival Crevicular Fluid) to the periodontal pocket. ${ }^{27}$ The IL- $1 \beta$ is immune cells mediator, which is released as bacterial product, such as LPS interact with TLRs. This cytokine increases neutrophil and expression of adhesion molecules and cause vascular modifications. When these cytokines are produced continuously, it can cause periodontal tissue damage. ${ }^{28}$

A study showed that there are significant differences in the concentrations of IL- $1 \beta$ in GCF between chronic periodontitis and control groups. The results showed that the concentration of IL- $1 \beta$ in GCF was higher in patients with periodontitis than in patients with healthy periodontium status. ${ }^{29}$ Application of capsicum frutescens $\mathrm{L}$ in periodontitis patients will provide meaningful effect, and a decrease in proinflammatory cytokine IL- $1 \beta$ so that the periodontal tissue damage can be suppressed and increase in TRPV-1 receptor to provide an analgesic effect. ${ }^{2,4}$

\section{Conclusion}

From this study, it can be concluded that the application of Capsicum frutescens $\mathrm{L}$ can lead to the increase of TRPV- 1 and decrease in IL- $1 \beta$, but does not affect the TLR-4. It means that there is an increase of pain receptors that will also increase the anti-inflammatory effect. The capsaicin can be used for healing or decreasing pain and accelerate healing in periodontitis. Suggestion for further research is to carry out research on the genetic detection of the pain in the periodontal tissues.

\section{Conflict of Interest}

The authors report no conflict of interest.

\section{References}

1. Ulbricht C, Seamon E. Natural Standard Herbal Pharmacotherapy. 2nd ed. Philadelphia: MOSBY Elsevier; 2010. p. 31.

2. Zhou Y, Guan X, Zhu W, et al. Capsaicin inhibits Porphyromonas gingivalis growth, biofilm formation, gingivomucosal, inflammatory cytokine secretion and in vitro osteoclastogenesis. Europ J Clin Microbiol and Infect Dis Springer Berlin 2014;1: 27-53.

3. Triska, Dewi HC, Khasanah LU, et al. Optimasi ekstraksi oleoresin cabai rawit hijau (capsicum frutescens L.) melalui metode maserasi. Jurnal Teknosains Pangan 2012;1: 1.

4. $\mathrm{Xu} \mathrm{Q}$, Barrios CA, Cutright $\mathrm{T}$, et al. Evaluation of toxicity of capsaicin, zosteric acid and their potential application as antifoulants. Environ Toxicol 2015;20: 467-474.

5. Demirbilek S, Ersoy MO, Karaman A, et al. Small-dose capsaicin reduces systemic inflammatory responses in septic rats. Anesth Analg 2004;99: 1501-1507.

6. Nagahama $\mathrm{M}$, Kihara $\mathrm{A}$, Kintoh $\mathrm{H}$, et al. Involvement of tumour necrosis factor- $\alpha$ in Clostridium perfringens $\beta$-toxin-induced-plasma extravasation in mice. $\mathrm{Br} \mathrm{J}$ Pharmacol 2008;153: 1296-1302.
7. Anand P, Bley K. Topical capsaicin for pain management: therapeutic potential and mechanisms of action of the new high-concentration capsaicin $8 \%$ patch. Br J Anaesth 2011;107: 490-502.

8. Sunariani J. Indera rasa pengecap di dalam rongga mulut. Surabaya: Dwiputra Pustaka Jaya; 2014. p. 57, 65, 90.

9. Lemeshow S, Davin WH-Jr. Besar sampel dalam penelitian kesehatan. Yogyakarta: Gadjahmada University Press.

10. Zhou Q, Desta T, Fentom M, et al. LPS cytokine profiling of macrophages exposed to porphyromonas gingivalis, its lipopolysaccharide, or its FimA protein. Infect Immun 2005;73: 935-942.

11. Aghaloo TL, Kang B, Sung EC, et al. Periodontal disease and bisphosphonates induced osteonecrosis of the jaws in the rat. J Bone Miner Res 2011;26: 1871-1882.

12. Soini Y, Paako P, LehtoVP. Histopathological evaluation of apoptosis in cancer. Am Jl of Pathy 1997;153: 1041-1048.

13. Pizem J, Cor A. Detection of apoptotic cells in tumour paraffin sections. Radiol Oncol 2003;37: 225-232.

14. O'Neill J, Brock C, Olesen AE, et al. Unravelling the mystery of capsaicin: a tool to understand and treat pain. Pharmacological Review 2012;64: 939-971.

15. Szallasi A, Blumbergn PM. Vanilloid (capsaicin) receptors and mechanism. Pharmacological reviews 1999;51: 3449,159-212.

16. Yang BH, Piao ZG, Kim YB, et al. Activation of vanilloid receptor 1 (VR1) by eugenol. J Dent Res 2003;82: 781-785.

17. Ramsey IS, Delling M, Clapham DE. An introduction to TRP channels. Annu Rev Physiol 2006;68: 619-647.

18. Gamse R, Petsche U, Lembeck F, et al. Capsaicin applied to peripheral nerve inhibits axoplasmic transport of substance P and somatostatin. Brain Res 2002: 239, 447462.

19. Hajishengallis G. Immunomicrobial pathogenesis of periodontitis: keystones, pathobionts, and host response. Trends Immunol 2014;35: 3-11.

20. Öztürk A, Yildiz L. Expression of transient receptor potential vanilloid receptor 1 and Toll-like receptor 4 in aggressive periodontitis and in chronic periodontitis. J Periodontal Res 2011;46: 475-482.

21. Miller SI, Ernst RK, Bader MW. LPS, TLR4 and infectious disease diversity. Nat Rev Microbiol 2005;3: 36-46.

22. Ferraz CCR, Diógenes A, Henry MA, et al. LPS from Porphyromonas gingivalis sensitizes capsaicin-sensitive nociceptors. J endo 2011;37: 45-48.

23. Teng YT. Protective and destructive immunity in the periodontium: Part 1-innate and humoral immunity and the periodontium. J Dent Res 2006;85: 198-208.

24. Reher VG, Zenobio EG, Costa FO, et al. Nitric oxide levels in saliva increase with severity of chronic periodontitis. J Oral Sci 2007;49: 271-276.

25. Gallin JI, Snyderman R, Fearon DT, et al. Inflammation basic principles and clinical correlates. 3 rd ed. Philadelphia. USA: Lippincott Williams \& Wilkins; 1999. p. 433-436, 443-451, 837, 1207-1211.

26. Giannopoulou C, Kamma JJ, Mombelli A. Effect of inflammation, smoking and stress on gingival crevicular fluid cytokine level. J Clin Periodontol 2003;30: 145-153.

27. Kinane DF, Lappin DF. Immune processes in periodontal disease: a review. Ann Periodontol 2002. p. 62-71.

28. Alwan AH. Determination of Interleukin-1b and Interleukin-6 in gingival crevicular fluid in patients with chronic periodontitis. J Dent \& Medical Sci 2015;14: 87.

29. Yoshinari N, Kawase H, Mitani A. Effects of scaling and root planing on the amounts of interleukin-1 and interleukin-1 receptor antagonist and the mRNA expression of interleukin-1 $\beta$ in gingival crevicular fluid and gingival tissues. J Periodont Res 2004;39: 158-167.

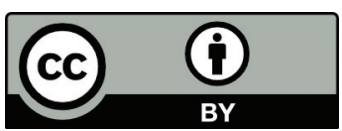

This work is licensed under a Creative Commons Attribution 